\title{
Pediatric Subungual Exostosis
}

\author{
Faraz Yousefian, DO; Brandon Davis, DO; John C. Browning, MD, MBA
}

\section{PRACTICE POINTS}

- Nail dystrophy can have a variety of causes, most commonly trauma, onychomycosis, verrucae, or subungual exostosis.

- Exostosis is a benign osteochondral tumor commonly found on the lateral or medial aspect of the hallux (great toe) in pediatric and young adult patients.

- A radiograph can be used as a preliminary tool for diagnosis, but subungual exostosis must be confirmed by biopsy or tissue histology at the time of excision.

Exostosis is a type of benign bone tumor in which trabecular (spongy) bone overgrows its normal border in a nodular pattern. When the growth occurs under the nail bed, it is termed subungual exostosis or Dupuytren exostosis. This condition may mimic other bony abnormalities such as an osteochondroma and may present with nail deformities with or without pain. For this reason, a biopsy of the lesion is necessary to rule out a precancerous growth. In rare cases, pediatric patients may have subungual exostosis, as demonstrated in our case.

Cutis. 2021;108:256-257.

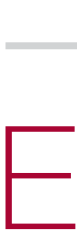

xostosis is a type of benign bone tumor in which trabecular (spongy) bone overgrows its normal border in a nodular pattern. ${ }^{1,2}$ Histologically, it usually is surrounded by a fibrocartilaginous cap. ${ }^{3}$ It is most commonly found on the lateral or medial aspect of the foot and is thought to be caused by trauma, either physical pressure or infection. ${ }^{4}$ When this lesion is found under the nail bed, it is termed subungual exostosis (Dupuytren exostosis). ${ }^{3}$ Sequelae of a subungual exostosis include nail dystrophy and lifting of the nail away from the toe, in addition to infection and possible loss of the toenail (onycholysis). There are only 2 genetic conditions related to exostosis: hereditary multiple exostosis and multiple exostoses-mental retardation syndrome.

An exostosis may appear to be a wart on first inspection. It may present similar to osteochondromas, and the only way to get a true diagnosis is by biopsy of the lesion. The treatment for an exostosis is surgery. The surgeon must remove the lesion at the base of the bone from which it grows to prevent recurrence of the lesion. ${ }^{5}$

Because exostosis may cause nail bed disruption, the differential diagnosis may include nail deformities, such as traumatic onycholysis, onychogryphosis, verrucae, subungual infection, or nail trauma. ${ }^{6,7}$

\section{Case Report}

A 7-year-old boy presented with changes of the right great toenail over the last 4 months. The patient noted that the affected nail was discolored, dystrophic, painful, and thickened. He did not recall prior trauma to the affected nail, and his mother stated that the lesion was growing and becoming more painful with a throbbing sensation at times. He described the pain as stabbing, which was exacerbated while walking and playing sports. Neither the patient nor his family had ever had any similar condition. He was not taking any medications, only a daily multivitamin. He had a history of eczematous dermatitis and keratosis pilaris without any other medical illnesses. He had a family history of psoriasis; however, no prior instances of exostosis had been reported. He had no medication allergies.

A full-body cutaneous and nail examination showed a well-developed, well-nourished boy who was in

Drs. Yousefian and Davis are from the University of the Incarnate Word School of Osteopathic Medicine, San Antonio, Texas. Dr. Browning is from Texas Dermatology and Laser Specialists, San Antonio.

The authors report no conflict of interest.

Correspondence: Faraz Yousefian, DO, University of the Incarnate Word School of Osteopathic Medicine, 7615-1 Kennedy Circle, San Antonio,

TX 78235 (Yousefian.faraz@gmail.com).

doi:10.12788/cutis.0380 


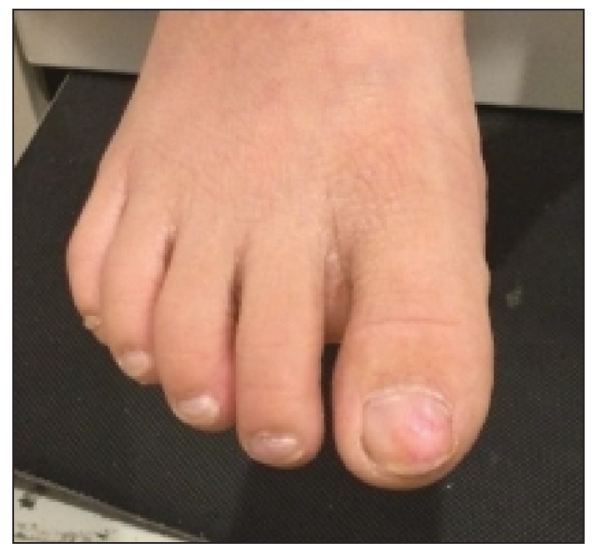

FIGURE 1. Subungual, pink, pearly nodule on the right great toe.

no acute distress. A firm, subungual, pink, pearly, hyperkeratotic nodule was appreciated on the right great toe (Figure 1). The lesion was tender to palpation. The rest of the examination and review of systems were normal.

From the clinical findings, a differential diagnosis of glomus tumor, hemangioma, and infection was considered. Periodic acid-Schiff stain was negative, which ruled out fungal infection. Nail avulsion and a shave biopsy were performed under general anesthesia. There was an exostosis arising from the dorsal aspect of the great toe measuring approximately $5 \mathrm{~mm}$ in width at the base and approximately $1 \mathrm{~mm}$ in height, which endorsed a diagnosis of distal phalanx subungual exostosis. A postsurgery radiograph (Figure 2) showed residual bone below the level of shave removal at the nail bed.

\section{Comment}

Exostosis is most commonly found on the lateral or medial aspect of the hallux (great toe) in patients younger than 18 years. ${ }^{8}$ Diagnosis often is obvious, even without a radiograph or biopsy, because the exostosis comes out from under the tip of the nail. Our case was interesting because the patient was a child, and the exostosis did not lift the nail or extrude from the distal tip of the nail bed. Evidence suggests that a greater-than-expected genetic influence contributes to an exostosis, though further investigation is needed to determine all of the causes and risk factors for subungual bony exostosis. Timely diagnosis and treatment are essential to the

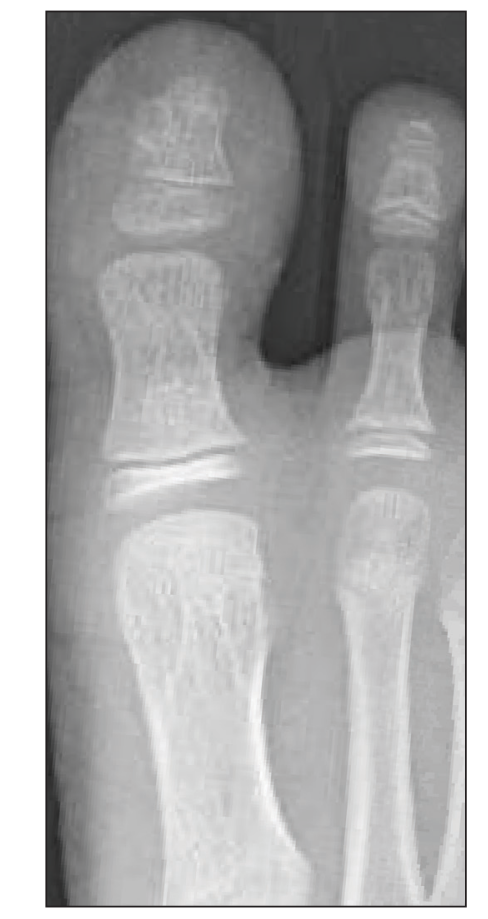

FIGURE 2. A radiograph of the right great toe showed softtissue changes.

prevention of sequelae of the disease, such as toe infection or chronic pain.

\section{REFERENCES}

1. de Palma L, Gigante A, Specchia N. Subungual exostosis of the foot. Foot Ankle Int. 1996;17:758-763. doi:10.1177/107110079601701208

2. Multhopp-Stephens H, Walling AK. Subungual (Dupuytren's) exostosis. J Pediatr Orthop. 1995;15:582-584. doi:10.1097/01241398 -199509000-00006

3. Davis DA, Cohen PR. Subungual exostosis: case report and review of the literature. Pediatr Dermatol. 1996;13:212-218.

4. Guarneri C, Guarneri F, Risitano G, et al. Solitary asymptomatic nodule of the great toe. Int J Dermatol. 2005;44:245-247.

5. Letts M, Davidson D, Nizalik E. Subungual exostosis: diagnosis and treatment in children. J Trauma. 1998;44:346-349.

6. Hoy NY, Leung AKC, Metelitsa AI, et al. New concepts in median nail dystrophy, onychomycosis, and hand, foot, and mouth disease nail pathology. ISRN Dermatol. 2012;2012:680163.

7. Rich P, Scher RK. Examination of the nail and work-up of nail conditions. In: Rich P, Scher RK, eds. An Atlas of Diseases of the Nail. Parthenon Publishing; 2003.

8. DaCambra MP, Gupta SK, Ferri-de-Barros F. Subungual exostosis of the toes: a systematic review. Clin Orthop Relat Res. 2014;472:1251-1259. doi:10.1007/s11999-013-3345-4 